\title{
Diagnóstico da tuberculose pulmonar em idosos de um hospital universitário no período 2009-2013, Belém, Pará
}

\author{
Emanuele Cordeiro Chaves*, Irna Carla do Rosário Souza Carneiro**, Maria Izabel Penha de Oliveira Santos***, \\ Nathália de Araújo Sarges ${ }^{\star \star \star *}$, Débora Suellen de Oliveira Guimarães ${ }^{\star \star \star \star *}$
}

\section{Resumo}

O processo de envelhecimento torna o idoso mais susceptível ao adoecimento por tuberculose, devido à imunossenescência, além das comorbidades frequentes nesse grupo etário, que tornam mais complexos o diagnóstico e o manejo clínico nos idosos. Dessa forma, objetivou-se avaliar os elementos de diagnóstico de tuberculose pulmonar em idosos atendidos no Hospital Universitário da Universidade Federal do Pará, Pará, referência em tuberculose pelo Ministério da Saúde. Trata-se de um estudo do tipo coorte retrospectivo, cuja amostra foi de 62 casos de tuberculose pulmonar em idosos diagnosticados no período entre 2009 e 2013. Houve concentração na faixa etária de 60-69 anos (56,5\%). Os principais sinais e sintomas foram febre $(69,4 \%)$, tosse produtiva $(67,7 \%)$, dispneia $(66,1 \%)$ e emagrecimento $(66,1 \%)$. Dentre os exa- mes laboratoriais, o de baciloscopia do escarro predominou (90,3\%), sendo positivo na maior parte dos casos. Os exames de imagem foram realizados na maioria dos idosos $(93,5 \%)$, sendo suspeito em grande parcela desses (98,3\%). Na relação da faixa de idade com a prova terapêutica, não se verificou significância estatística $(p=0,4318)$. O diagnóstico da tuberculose no idoso apresenta especificidades, fazendo-se necessário o fortalecimento de estratégias de cuidado na Atenção Primária à Saúde.

Palavras-chave: Diagnóstico. Idoso. Tuberculose pulmonar.

* Enfermeira. Mestra em Doenças Tropicais pelo Núcleo de Medicina Tropical, Universidade Federal do Pará, Belém, Pará, Brasil. Endereço para correspondência: Av. Conselheiro Furtado, 1734, CEP: 66040-100, Cremação, Belém, Pará. E-mail: manu.chaves@hotmail.com

** Médica. Doutora em Doenças Infecciosas e Parasitárias pela Universidade Federal de São Paulo. Docente do Programa de Pós-Graduação em Doenças Tropicais, Núcleo de Medicina Tropical, Universidade Federal do Pará. E-mail: irnajula@uol.com.br

*** $\quad$ Enfermeira. Doutora em Enfermagem pela Universidade Federal do Rio de Janeiro. Docente do Mestrado em Enfermagem, Universidade do Estado do Pará e Universidade Federal do Amazonas. E-mail: princesa50@ hotmail.com

**** Enfermeira. Mestra em Enfermagem pelo Mestrado Associado da Universidade do Estado do Pará e Universidade Federal do Amazonas. E-mail: nathdream@hotmail.com

****** Enfermeira. Mestra em Doenças Tropicais pelo Núcleo de Medicina Tropical, Universidade Federal do Pará. E-mail: debora.ipb@uol.com.br

$\rightarrow$ http://dx.doi.org/10.5335/rbceh.v13i2.6558

Recebido em: 19.11.2016. Aceito em: 20.03.2017. 


\section{Introdução}

A tuberculose é uma doença infecciosa bacteriana, de evolução crônica, que compromete principalmente os pulmões, sendo causada pelo Mycobacterium tuberculosis e transmitida predominantemente por via aérea (SANTOS et al., 2013; MELO et al., 2009). Segundo a Organização Mundial da Saúde (OMS), estima-se que $1 / 3$ da população do mundo seja infectada pelo $M$. tuberculosis, e no Brasil foram notificados 68 mil casos novos de tuberculose em 2014 (BRASIL, 2015).

No Brasil, a transição epidemiológica ocorre de forma "atípica", caracterizando-se tanto pela reemergência ou presença constante das doenças infecciosas e parasitárias quanto pela maior prevalência de doenças e agravos crônicos não transmissíveis (PEREIRA; ALVES-SOUZA; VALE, 2015).

Com o envelhecimento, ocorre frequentemente a perda de reatividade imune celular para o Mycbacterium tuberculosis, o que torna os indivíduos mais vulneráveis tanto para a infecção exógena quanto para a reativação de focos contendo bacilos em estado de latência (HUSSEIN; YOUSSEF; ABUSEDERA, 2013).

Além disso, estudos afirmam que a doença se comporta de forma diferenciada entre os mais jovens e os mais velhos, por isso, ambos devem ser avaliados de forma diferenciada e, quando essas singularidades são ignoradas, o estabelecimento de um diagnóstico pode ser adiado, levando a um aumento da morbidade e da mortalidade nos idosos (KWON et al., 2013; PARDESHI; DESMUKH, 2007). Nos idosos, os sintomas da doença, geralmente, são difíceis de aferir pela frequente concomitância de doenças respiratórias, cardiovasculares e sistêmicas, que apresentam quadro clínico semelhante, associado à dificuldade de o idoso relatar as queixas, devido ao déficit de memória, a estados confusionais, à senilidade e a problemas na verbalização, o que resulta na demora do diagnóstico (CANTALICE FILHO; SANT'ANNA; BÓIA, 2007).

Apesar de ser uma causa evitável de morte em pacientes idosos, a tuberculose apresenta taxa de mortalidade seis vezes maior entre esse grupo etário, provavelmente, tal situação se deva à imunossupressão relacionada com idade, desnutrição, comorbidades e diagnóstico tardio (HUSSEIN; YOUSSEF; ABUSEDERA, 2013). Destaca-se que o retardo no diagnóstico da tuberculose entre os idosos é um dos problemas que acentua a gravidade da doença e pode decorrer da dificuldade de acesso ao serviço de saúde, do acolhimento inadequado do cliente, da baixa prioridade na procura de sintomas respiratórios, de contatos intradomiciliares e de baixo nível de suspeição diagnóstica de tuberculose (OLIVEIRA et al., 2013).

Dessa forma, o objetivo deste estudo foi avaliar os elementos de diagnóstico de tuberculose pulmonar em idosos de um hospital universitário no período 20092013 em Belém, PA, com propósito de levantar subsídios que contribuam para a melhoria na qualidade da assistência 
gerontológica prestada pela equipe interdisciplinar em doenças transmissíveis, especialmente no que concerne à tuberculose em idosos.

\section{Métodos}

Trata-se de um estudo epidemiológico do tipo coorte retrospectivo. A pesquisa foi realizada no Hospital Universitário João de Barros Barreto, da Universidade Federal do Pará (UFPA), Belém, PA, utilizando prontuários de casos de tuberculose diagnosticados no período 2009-2013. Adotou-se como critérios de inclusão ter idade igual ou superior a 60 anos, ser caso novo ou recidivo ou reingresso após abandono de tuberculose pulmonar e ter iniciado o tratamento na instituição em que foi realizada a pesquisa. Utilizou-se como critérios de exclusão prontuários incompletos e com encerramento do caso como mudança de diagnóstico. A amostra final do estudo foi composta por 62 idosos, cujos dados foram obtidos no prontuário hospitalar.

Para a análise dos dados, foi criado um banco no programa eletrônico BioEstat versão $5.0 \mathrm{e}$, posteriormente, os dados foram analisados por meio da aplicação do Teste $\mathrm{G}$, admitindo-se nível $\alpha=0,05$ (5\%) e valor de $\mathrm{P} \leq 0,05$.

$O$ estudo foi realizado em concordância com a Resolução no 466/2012, do Conselho Nacional de Saúde, que dispõe sobre os aspectos éticos envolvendo a pesquisa com seres humanos. Nesse sentido, o projeto foi aprovado (Parecer no 1.081.347) pelo Comitê de Ética em Pesquisa do Núcleo de Medicina Tropical da UFPA.

\section{Resultados}

Houve predominância do sexo masculino, a média de idade foi de $69,8( \pm 7,1)$ anos, com concentração na faixa etária de 60-69 anos. A maioria dos idosos apresentaram escolaridade de ensino fundamental completo ou incompleto e eram oriundos de Belém ou da região metropolitana (Tabela 1).

Tabela 1 - Dados sociodemográficos dos idosos com tuberculose pulmonar - Belém, PA - 2009-2013

\begin{tabular}{l|l|r}
\cline { 2 - 3 } \multicolumn{1}{c|}{} & \multicolumn{1}{|c}{ Variáveis } & Representação \\
\hline Sexo & Masculino & $41(66,1 \%)$ \\
& Feminino & $21(33,9 \%)$ \\
\hline Faixa etária & $60-69$ anos & $35(56,5 \%)$ \\
& $70-79$ anos & $22(35,5 \%)$ \\
& $80-89$ anos & $2(3,2 \%)$ \\
& $\geq 90$ anos & $3(4,8 \%)$ \\
\hline Grau de & Analfabeto & $7(11,3 \%)$ \\
instrução & Fundamental & $44(71,0 \%)$ \\
& completo/ & \\
& incompleto & $4(6,5 \%)$ \\
& Médio completo/ & \\
& incompleto & $7(11,3 \%)$ \\
\hline Município de & Não há registro & Belém e região \\
origem & metropolitana & $46(74,2 \%)$ \\
& Interior do estado & $15(24,2 \%)$ \\
& Outro estado & $1(1,6 \%)$ \\
\hline
\end{tabular}

Fonte: prontuários dos pacientes.

Os principais sinais e sintomas apresentados pelos idosos com tuberculose foram febre $(43 / 62 ; 69,4 \%)$, tosse produtiva $(42 / 62 ; 67,7 \%)$, dispneia $(41 / 62 ; 66,1 \%)$ e emagrecimento $(41 / 62 ; 66,1 \%)$. Em menor proporção, foram relatados dor torácica $(30 / 62 ; 48,4 \%)$, escarros hemoptoicos $(17 / 62 ; 27,4 \%)$, fadiga $(17 / 62 ; 27,4 \%)$, hiporexia $(15 / 62 ; 24,2 \%)$, tosse seca $(14 / 62$; $22,6 \%)$ e sudorese $(8 / 62 ; 12,9 \%)$. 
Em relação aos elementos utilizados para o diagnóstico, o exame laboratorial de baciloscopia do escarro predominou, sendo positivo na maior parte dos casos.
Os exames de imagem foram realizados em percentual considerável dos idosos e teve resultado suspeito na maioria deles (Tabela 2).

Tabela 2 - Dados de diagnóstico dos idosos com tuberculose pulmonar, Belém, PA - 2009-2013

\begin{tabular}{|c|c|c|}
\hline & Variáveis & Representação \\
\hline Baciloscopia do escarro & $\begin{array}{l}\text { Realizou } \\
\text { Não realizou }\end{array}$ & $\begin{array}{r}56(90,3 \%) \\
6(9,7 \%)\end{array}$ \\
\hline Resultado da baciloscopia do escarro* & $\begin{array}{l}\text { Positiva } 1 \text { amostra } \\
\text { Positiva } 2 \text { amostras } \\
\text { Negativa } 1 \text { amostra } \\
\text { Negativa } 2 \text { amostras }\end{array}$ & $\begin{array}{r}24(42,8 \%) \\
17(30,3 \%) \\
2(3,6 \%) \\
13(23,2 \%) \\
\end{array}$ \\
\hline Baciloscopia de outro material $^{* *}$ & $\begin{array}{l}\text { Realizou } \\
\text { Não realizou }\end{array}$ & $\begin{array}{l}12(19,4 \%) \\
50(80,6 \%)\end{array}$ \\
\hline Resultado da baciloscopia de outro material ${ }^{*, \star *}$ & $\begin{array}{l}\text { Positiva } \\
\text { Negativa }\end{array}$ & $\begin{array}{l}4(33,3 \%) \\
8(66,7 \%)\end{array}$ \\
\hline Cultura do escarro & $\begin{array}{l}\text { Realizou } \\
\text { Não realizou }\end{array}$ & $\begin{array}{l}10(16,1 \%) \\
52(83,9 \%)\end{array}$ \\
\hline Resultado da cultura do escarro* & $\begin{array}{l}\text { Positiva } \\
\text { Negativa }\end{array}$ & $\begin{array}{l}9(90,0 \%) \\
1(10,0 \%) \\
\end{array}$ \\
\hline Cultura de outro material ${ }^{\star \star}$ & $\begin{array}{l}\text { Realizou } \\
\text { Não realizou }\end{array}$ & $\begin{array}{r}3(4,8 \%) \\
59(95,2 \%) \\
\end{array}$ \\
\hline Resultado da cultura de outro material ${ }^{\star, \star *}$ & $\begin{array}{l}\text { Positiva } \\
\text { Negativa }\end{array}$ & $\begin{array}{r}3(100,0 \%) \\
0(0,0 \%) \\
\end{array}$ \\
\hline Histopatológico & $\begin{array}{l}\text { Realizou } \\
\text { Não realizou }\end{array}$ & $\begin{array}{r}3(4,8 \%) \\
59(95,2 \%) \\
\end{array}$ \\
\hline Resultado do histopatológico* & $\begin{array}{l}\text { BAAR sugestivo } \\
\text { Sugestivo de tuberculose } \\
\text { Não sugestivo }\end{array}$ & $\begin{array}{l}1(33,3 \%) \\
1(33,3 \%) \\
1(33,3 \%)\end{array}$ \\
\hline Exames radiológicos & $\begin{array}{l}\text { Realizou } \\
\text { Não realizou }\end{array}$ & $\begin{array}{r}58(93,6 \%) \\
4(6,5 \%) \\
\end{array}$ \\
\hline Resultado dos exames radiológicos* & $\begin{array}{l}\text { Suspeito } \\
\text { Normal }\end{array}$ & $\begin{array}{r}57(98,3 \%) \\
1(1,7 \%)\end{array}$ \\
\hline Prova tuberculínica & $\begin{array}{l}\text { Realizou } \\
\text { Não realizou }\end{array}$ & $\begin{array}{l}22(35,5 \%) \\
40(64,5 \%)\end{array}$ \\
\hline Resultado da prova tuberculínica ${ }^{\star, \star \star \star}$ & $\begin{array}{l}\text { Não reator } \\
\text { Reator fraco } \\
\text { Reator forte }\end{array}$ & $\begin{array}{r}9(40,9 \%) \\
2(9,1 \%) \\
11(50,0 \%) \\
\end{array}$ \\
\hline
\end{tabular}

Fonte: prontuários dos pacientes.

Nota: *Considerando apenas os pacientes que realizaram o exame; **Lavado broncoalveolar; *** Classificação vigente no período do estudo. 
$\mathrm{Na}$ relação da faixa de idade com a prova terapêutica, verificou-se que a maioria dos idosos que realizaram a prova terapêutica, assim como os que não realizaram, estavam concentrados na faixa etária de 60-69 anos, não havendo significância estatística nessa relação (Tabela 3).

Tabela 3 - Distribuição dos idosos com tuberculose pulmonar quanto à faixa de idade e à realização de prova terapêutica, Belém, PA - 2009-2013

\begin{tabular}{l|c|c|c|c}
\cline { 2 - 4 } & \multirow{2}{*}{ Variáveis } & $\begin{array}{c}\text { Realizou prova } \\
\text { terapêutica }\end{array}$ & $\begin{array}{c}\text { Não realizou prova } \\
\text { terapêutica }\end{array}$ & \multirow{2}{*}{${ }^{*} p$} \\
\cline { 2 - 4 } Exame & Representação & Representação & \\
\hline Faixa de idade & Prova terapêutica & $14(22,6 \%)$ & $48(77,4 \%)$ & \\
& $60-69$ anos & $9(64,3 \%)$ & $26(54,2 \%)$ & 0,4318 \\
& $70-79$ anos & $5(35,7 \%)$ & $17(35,4 \%)$ & \\
& $80-89$ anos & $0(0,0 \%)$ & $2(4,2 \%)$ & \\
& $\geq 90$ anos & $0(0,0 \%)$ & $3(6,3 \%)$ & \\
\hline
\end{tabular}

Fonte: prontuários dos pacientes.

Nota: * $p$ valor $\leq 0,05$. Teste $\mathrm{G}$.

\section{Discussão}

A maioria dos idosos pertencia ao sexo masculino, semelhante ao identificado em outros estudos envolvendo idosos hospitalizados (STORTI et al., 2013; SASS; MARCON, 2012). O predomínio de homens hospitalizados pode ser explicado pelo fato de que os indivíduos do sexo masculino são menos cuidadosos com a própria saúde, estando, dessa forma, mais propensos à necessidade de internação hospitalar (STORTI et al., 2013).

Além disso, a tuberculose é uma doença que, historicamente, afeta mais homens, em todas as faixas etárias. Segundo dados do Ministério da Saúde, $66,8 \%$ dos casos de tuberculose registrados em 2014 foram entre homens, percentual muito próximo ao encontrado neste estudo (BRASIL, 2015).
Estudos realizados no Irã e na Coréia do Sul demonstraram idade média dos pacientes $(69,6$ anos e 72 anos respectivamente) muito próxima àquela do presente estudo (69,8 anos) (KWON et al., 2013; TOWHIDI; AZARIAN; ASNAASHARI, 2008). No Recife, em pesquisa semelhante, a idade média dos idosos foi de 69 anos (CAVALCANTI et al., 2006).

Quanto ao grau de instrução, a maioria dos idosos apresentou ensino fundamental incompleto ou completo. Em se tratando de tuberculose, a escolaridade é um fator de extrema relevância, já que o analfabetismo e a baixa escolaridade relacionam-se com maior probabilidade de abandonar o tratamento, devido à menor compreensão e ao acesso restrito desses indivíduos a informações sobre a doença. Dessa forma, cabe ao profissional de saúde oferecer aos pacientes 
informações claras sobre a doença e o tratamento (FURLAN; OLIVEIRA; MARCON, 2012).

Quanto ao município de origem, a maioria dos idosos, de ambos os sexos, eram oriundos da região metropolitana de Belém, PA, o que já era esperado, tendo em vista que essa região é a que mais concentra o número de casos de tuberculose no estado. A tuberculose, de forma geral, concentra-se nos centros urbanos, devido ao fato de ter sua distribuição influenciada por fatores como a extensão territorial, o crescimento populacional desordenado e a concentração de pessoas nas periferias (QUEIROGA et al., 2012).

Em relação aos achados clínicos, a ocorrência de febre foi a manifestação mais relatada pelos idosos $(69,4 \%)$. Contudo, um estudo realizado no Rio de Janeiro, que comparou as manifestações clínicas da tuberculose em idosos e não idosos, identificou que a presença de febre foi mais comum no segundo grupo $(69,3 \%)$ do que no primeiro $(55,4 \%)$ (CANTALICE FILHO; SANT'ANNA; BÓIA, 2007).

No México, em período de 12 anos de estudo, foi evidenciado que os indivíduos mais velhos eram menos propensos a apresentar febre, o que pode ser explicado pela possível redução da resposta do centro termorregulador do hipotálamo às prostaglandinas $\mathrm{E} 2$ ou uma maior sensibilidade ao centro de alfa-MSH, que pode contribuir para a redução da resposta febril com o avançar da idade (HERVERT et al., 2012).

A presença de tosse produtiva $(67,7 \%)$ foi superior em relação à de tosse seca
$(22,6 \%)$, o que ratifica o diagnóstico tardio dos idosos, tendo em vista que, geralmente, a doença inicia-se com tosse seca, agravando-se ao longo dos dias, podendo evoluir para tosse com expectoração (PINHEIRO, 2015). Portanto, no presente estudo, evidenciou-se que, apesar de a literatura relatar menor frequência de tosse, devido à redução do reflexo com o envelhecimento, ainda assim, ela constitui um indicador importante para o diagnóstico da doença (CAVALCANTI et al., 2006). Contudo, esse sintoma pode ter sido influenciado por presença de doenças de base ou tabagismo.

A dispneia foi identificada em $66,1 \%$ dos idosos, representando percentual consideravelmente superior ao relatado em outros estudos, envolvendo tanto esse grupo etário quanto a população em geral (KWON et al., 2013; CANTALICE FILHO; SANT'ANNA; BÓIA, 2007). Tal achado indica que a maioria dos idosos do presente estudo foi hospitalizada em um curso tardio da doença e em estado bastante agravado, já que a dispneia, geralmente, ocorre quando há comprometimento de uma grande área pulmonar (NOGUEIRA et al., 2006).

$\mathrm{O}$ emagrecimento foi relatado por $66,1 \%$ dos pacientes. A perda de peso no idoso com tuberculose apresenta resultados controversos na literatura, por exemplo, estudo realizado no Rio de Janeiro identificou essa manifestação clínica em $79,1 \%$ dos idosos, enquanto que em pesquisa realizada na Coréia do Sul apenas $3 \%$ dos idosos apresentaram essa queixa (KWON et al., 2013; CANTALICE FILHO; SANT'ANNA; BÓIA, 
2007). Isso pode estar associado ao tempo de existência e extensão da doença, já que são proporcionais à perda de peso (MELO et al., 2009).

Além disso, a dispneia pode levar à perda de peso, provavelmente decorrente da incapacidade de ingerir quantidades suficientes de alimentos, os quais são necessários em maior quantidade devido à existência de um estado hipermetabólico ocasionado tanto pelo custo elevado de respiração como pela própria existência da doença (NOGUEIRA et al., 2006).

Quanto aos elementos de diagnóstico, a baciloscopia do escarro foi o exame laboratorial mais solicitado $(90,3 \%)$, sendo, ainda hoje, o método mais importante em um programa de descoberta e controle dos casos de tuberculose pulmonar e laríngea, dela dependendo o diagnóstico etiológico, a orientação e o controle do tratamento (BRASIL, 2014).

Dentre os que realizaram o exame de baciloscopia, a positividade foi de $42,8 \%$ para uma amostra e $30,3 \%$ para duas amostras de escarro, contudo, considerando a positividade total, esse percentual é de $73,1 \%$, próximo ao relatado em estudo semelhante, no qual a positividade desse exame foi de $65,1 \%$ para os idosos e não se verificou diferença estatisticamente significativa entre os resultados de idosos e não idosos (CANTALICE FILHO; SANT'ANNA; BÓIA, 2007).

Quanto à cultura do escarro, apenas $16,1 \%$ dos idosos realizaram esse exame, o que pode estar relacionado tanto à dificuldade da coleta do material quanto ao direcionamento do Ministério da Saúde quanto à obrigatoriedade de exame de cultura ser apenas para casos de retratamento e para populações mais vulneráveis (indígenas, pessoas em situação de rua, privadas de liberdade e portadores de HIV/Aids) (BRASIL, 2011).

Entretanto, a positividade do exame de cultura do escarro foi de $90,0 \%$ dentre os idosos que o realizaram, indicando um percentual de sensibilidade elevado, evidenciado em outros estudos que não verificam diferença significativa entre os resultados desse exame entre idosos e não idosos (KWON et al., 2013; HAUER et al., 2011). Contudo, é necessário avaliar cuidadosamente tal informação, tendo em vista que essa pode indicar diagnóstico tardio, com a doença em estágio mais agravado nos grupos estudados (CANTALICE FILHO; SANT'ANNA; BÓIA, 2007).

$\mathrm{O}$ exame radiológico foi realizado em 93,5\% dos casos, apresentando resultado suspeito para tuberculose em $98,3 \%$ daqueles que o realizaram. Tal exame, no caso da tuberculose pulmonar, permite evidenciar infiltrados com cavitação nos lobos superior e médio do pulmão, sugerindo a presença da doença, contudo, isoladamente não é suficiente para o diagnóstico, e nos idosos pode apresentar-se de forma atípica, havendo maior ocorrência no lobo inferior do pulmão (KNECHEL et al., 2009). No entanto, devido ao fato de o presente estudo ter sido realizado em um serviço de referência, os profissionais com expertise na área puderam identificar a maioria dos casos suspeitos, mesmo com a possível apresentação atípica. 
A prova tuberculínica foi realizada em $35,5 \%$ dos idosos, apresentando resultado reator fraco em $9,1 \%$ e reator forte em $50 \%$ dos casos, somando uma positividade total de $59,1 \%$, valor próximo ao identificado em outro estudo (CANTALICE FILHO; SANT'ANNA; BÓIA, 2007). Apesar de ser um tema controverso na literatura, a realização desse exame nos idosos é importante, pois se trata de um método auxiliar no acompanhamento dos suspeitos e no consequente diagnóstico da doença (BRASIL, 2011).

O início do tratamento, por meio de prova terapêutica, ocorreu em 22,6\% dos casos, ou seja, foram casos que iniciaram tratamento sem confirmação laboratorial, baseados principalmente em achados clínicos, resultados de exames radiológicos e prova tuberculínica. A realização de prova terapêutica não se mostrou relacionada a alguma faixa de idade específica dentre os idosos estudados, contudo, representa um desafio para o profissional de saúde, que se vê diante do impasse entre o início do tratamento precoce e a consequente redução dos danos causados pela doença ou o início de um tratamento inadequado, que pode ocasionar reações adversas graves, já que se devem considerar as alterações de farmacodinâmica e farmacocinética inerentes ao envelhecimento, as comorbidades existentes e a polifarmácia.

As principais limitações do estudo decorrem do fato de a coleta de dados ter sido realizada de forma retrospectiva e documental, o que gerou viés de informação, com algumas perdas de registros.
São necessários, portanto, estudos de caso controle ou grandes coortes para melhor entender as peculiaridades da tuberculose na população idosa.

\section{Conclusão}

Neste estudo, verificou-se que houve predominância do sexo masculino, da faixa etária de idosos jovens, que apresentaram como principais achados clínicos febre, tosse produtiva, dispneia e emagrecimento. Quanto ao diagnóstico laboratorial, predominou a realização de baciloscopia do escarro, a qual foi positiva em percentual considerável dos idosos. Os exames de imagem foram utilizados em maioria absoluta dos casos, tendo como resultado predominante $o$ sugestivo de tuberculose. A realização de prova terapêutica foi elevada, o que indica que o diagnóstico da tuberculose em idosos é complexo e nem sempre pode ser confirmado por meio de exames laboratoriais, exigindo do médico a indicação de prova terapêutica.

Os resultados do presente estudo contribuem significativamente para a atenção à saúde do idoso, principalmente, no sentido de atentar à complexidade do diagnóstico da tuberculose, por vezes verificada nesse grupo etário, devido às suas especificidades, e à necessidade de melhor qualificação dos profissionais da Atenção Primária à Saúde, para que se realize o diagnóstico oportuno dos casos e, consequentemente, evite-se a internação para uma doença que deve ser tratada no nível primário de atenção. 


\section{Diagnosis of pulmonary tuberculosis in elderly patients at a university hospital in the period 2009-2013, Belém, Pará}

\section{Abstract}

The aging process makes the elderly more susceptible to tuberculosis due to immunosenescence, in addition to the frequent comorbidities in this age group, which make the diagnosis and clinical management of this aggravation more complex in the elderly. The objective of this study was to evaluate the diagnostic features of pulmonary tuberculosis in the elderly attended in the university hospital of the UFPA, Pará, a reference in tuberculosis by the Ministry of Health. This is a retrospective cohort study, which included 62 cases of tuberculosis Pulmonary in the elderly diagnosed in the period between 2009 to 2013. There was concentration in the age group of $60-69$ years $(56.5 \%)$. The main signs and symptoms were fever $(69.4 \%)$, productive cough $(67.7 \%)$, dyspnea $(66.1 \%)$ and weight loss $(66.1 \%)$. Among the laboratory tests, sputum smear microscopy predominated $(90.3 \%)$, being positive in most cases. Image exams were performed in the majority of the elderly $(93.5 \%)$, being suspected in a large number of these $(98.3 \%)$. In the relation of the age range with the therapeutic test, there was no statistical significance $(p$ $=0.4318$ ) . The diagnosis of tuberculosis in the elderly presents specificities, making it necessary to strengthen care strategies in Primary Health Care.

Keywords: Diagnosis. Aged. Pulmonary Tuberculosis.

\section{Referências}

BRASIL. Ministério da Saúde. Secretaria de Vigilância à Saúde. Programa Nacional de Controle da Tuberculose. Brasília: Ministério da Saúde, 2015. Disponível em: <https:// drive.google.com/file/d/0B0CE2wqdEaR-WUJ0RWZPcG0zM00/view>. Acesso em: 21 nov. 2015.

Ministério da Saúde. Secretaria de Vigilância em Saúde. Departamento de Vigilância Epidemiológica. Manual de recomendações para o controle da tuberculose. Brasília: Ministério da Saúde, 2011. 284 p. (Série A. Normas e Manuais Técnicos).

Ministério da Saúde. Secretaria de Vigilância em Saúde. Programa Nacional de Controle da Tuberculose. Teste Rápido Molecular para tuberculose (TRM-TB). Brasília: Ministério da Saúde, 2014.

CANTALICE FILHO, J. P.; SANT'ANNA, C. C.; BÓIA, M. N. Aspectos clínicos da tuberculose pulmonar em hospital universitário do Rio de Janeiro, RJ, Brasil. Jornal Brasileiro de Pneumologia, Brasília, v. 33, n. 6, p. 699-706, nov./dez. 2007.

CAVALCANTI, Z. R. et al. Características da tuberculose em idosos no Recife (PE): contribuição para o programa de controle. Jornal Brasileiro de Pneumologia, Brasília, v. 32, n. 6, p. 535-543, 2006.

FURLAN, M. C. R.; OLIVEIRA, S. P.; MARCON, S. S. Fatores associados ao abandono do tratamento de tuberculose no estado do Paraná. Acta Paulista de Enfermagem, São Paulo, v. 25, p. 108-114, 2012.

HAUER, B. et al. Tuberculosis in the elderly in Germany. European Respiratory Journal, Paris, v. 38, n. 2, p. 467-470, ago. 2011.

HERVERT, L. P. C. et al. Tuberculosis in ageing: high rates, complex diagnosis and poor clinical outcomes. Age Ageing, Oxford, v. 41, p. $488-495,2012$. 
HUSSEIN, M. T.; YOUSSEF, L. M.; ABUSEDERA, M. A. Pattern of pulmonar tuberculosis in elderly patients in Sohag Governorate: hospital based study. Egyptian Journal of Chest Diseases and Tuberculosis, Cairo, v. 62 , n. 2, p. 269-274, 2013.

KNECHEL, N. A. et al. Tuberculosis: pathophysiology, clinical features, and diagnosis. Critical Care Nurse, Columbia, v. 29, n. 2, p. 34-43, abr. 2009.

KWON, Y. S. et al. Clinical characteristics and treatment outcomes of tuberculosis in the elderly: a case control study. BMC Infectious Disease, Londres, v. 13, n. 121, p. 1-7, 2013.

MELO, F. A. et al. Tuberculose. In: FOCACCIA, R. et al. (Ed.). Veronesi: tratado de infectologia. 4. ed. São Paulo: Atheneu, 2009. p. 1263-1300.

NOGUEIRA, C. R. et al. Aspectos antropométricos, bioquímicos e sintomatológicos em mulheres com tuberculose pulmonar. Revista de Ciências Médicas, Campinas, v. 15, n. 4, p. 281-288, 2006.

OLIVEIRA, A. A.V. et al. Diagnóstico da tuberculose em pessoas idosas: barreiras de acesso relacionadas ao serviço de saúde. Revista da Escola de Enfermagem da USP, São Paulo, v. 47, n. 1, p. 145-151, 2013.

PARDESHI, G.; DESMUKH, D. Disease characteristics and treatment outcome in elderly tuberculosis patients on DOTS. Indian Journal of Community Medicine, Nova Délhi, v. 32, n. 4, p. 292-294, 2007.

PEREIRA, R. A.; ALVES-SOUZA, R. A.; VALE, J. S. O processo de transição epidemiológica: uma revisão de literatura. Revista Científica da Faculdade de Educação e Meio Ambiente, Porto Velho, v. 6, n. 1, p. 99-108, jan./jun. 2015.

PINHEIRO, P. 10 sintomas da tuberculose. 2015. Disponível em: <http://www.mdsaude. com/2012/07/sintomas-da-tuberculose.html>. Acesso em: 10 jan. 2016.
QUEIROGA, R. P. F. et al. Distribuição espacial da tuberculose e a relação com condições de vida na área urbana do município de Campina Grande - 2004 a 2007. Revista Brasileira de Epidemiologia, Brasília, v. 15, n. 1, p. 222-232, 2012.

SANTOS, J. R. P. et al. Tuberculose. In: LEÃO, R. N. Q. et al. (Ed.). Medicina tropical e infectologia na Amazônia. Belém: Samaúma, 2013. p. 1101- 1136.

SASS, A.; MARCON, S. S. Dependência para alimentar-se e consumo alimentar em idosos hospitalizados. Revista Brasileira de Enfermagem, Brasília, v. 65, n. 6, p. 955-61, 2012.

STORTI, L. B. et al. Fragilidade de idosos internados na clínica médica da unidade de emergência de um hospital geral terciário. Texto Contexto Enfermagem, Florianópolis, v. 22 , n. 2 , p. $452-9,2013$.

TOWHIDI, M.; AZARIAN, A.; ASNAASHARI, A. Pulmonary tuberculosis in elderly. Tanaffos, Teerã, v. 7, n. 1, p. 52-57, 2008. 